\title{
Reduction of 4-(haloalkyl)azetidin-2-ones with $\mathrm{LiAlH}_{4}$ as a powerful method for the synthesis of stereodefined aziridines and azetidines
}

\author{
Willem Van Brabandt, Yves Dejaegher, Robin Van Landeghem \\ and Norbert De Kimpe* \\ Department of Organic Chemistry, Faculty of Bioscience Engineering, \\ Ghent University, Coupure links 653, B-9000 Ghent, Belgium
}

norbert.dekimpe@UGent.be

\section{General experimental methods}

${ }^{1} \mathrm{H}$ NMR spectra were recorded at $300 \mathrm{MHz}$ with $\mathrm{CDCl}_{3}$ as solvent and tetramethylsilane as internal standard. ${ }^{13} \mathrm{C}$ NMR spectra were recorded at $75 \mathrm{MHz}$ with $\mathrm{CDCl}_{3}$ as solvent. Mass spectra were obtained with a mass spectrometer $(70 \mathrm{eV})$ using a GC-MS coupling (20 m glass capillary column, i.d. $0.53 \mathrm{~mm}$, He carrier gas) or were recorded using a direct inlet system $(70 \mathrm{eV})$. Diethyl ether was dried by distillation over sodium benzophenone ketyl. All other solvents were used as received from the supplier.

\footnotetext{
* Tel: +0032 92645951, fax: +003292646243
} 


\section{Synthesis of 4-(1-haloalkyl)azetidin-2-ones 1 and 4-(2-haloalkyl)azetidin-2-ones 11}

4-(1-Haloalkyl)azetidin-2-ones 1 and 4-(2-haloalkyl)azetidin-2-ones 11 were synthesised by [2+2]-cyclocondensation of the corresponding halogenated imines and ketenes (formed in situ from the corresponding acid chlorides by treatment with triethylamine) in benzene. Yields of 4-(1-haloalkyl)azetidin-2-ones 1 ranged from 73 to $85 \%$ while yields

of 4-(2-haloalkyl)azetidin-2-ones 11 were situated between 69 and 90\%. ${ }^{28,29}$ The spectroscopic data of compounds $\mathbf{1 b}$ and $\mathbf{1 1 a}$ are representative examples of these novel $\beta$-lactams.

\section{1-Benzyl-3-benzyloxy-4-(1-chloro-1-methylethyl)azetidin-2-one 1b}

White crystals, $73 \%$ yield, TLC Rf 0.35 (petroleum ether/ethyl acetate $3 / 1$ ), Mp: $37-39^{\circ} \mathrm{C}$ ${ }^{1} \mathbf{H}$ NMR (300 MHz, $\left.\mathrm{CDCl}_{3}\right): \delta 1.65$ and $1.73(6 \mathrm{H}, 2 \times \mathrm{s}), 3.90(1 \mathrm{H}, \mathrm{d}, J=5.2 \mathrm{~Hz}), 4.32$ $(1 \mathrm{H}, \mathrm{d}, J=14.6 \mathrm{~Hz}), 4.58(1 \mathrm{H}, \mathrm{d}, J=5.2 \mathrm{~Hz}), 4.67(1 \mathrm{H}, \mathrm{d}, J=11.8 \mathrm{~Hz}), 4.88(1 \mathrm{H}, \mathrm{d}, J=$ 14.6 Hz), $4.91(1 \mathrm{H}, \mathrm{d}, J=11.8 \mathrm{~Hz}), 7.22-7.37(5 \mathrm{H}, \mathrm{m}) .{ }^{13} \mathbf{C} \mathbf{N M R}\left(75 \mathrm{MHz}, \mathrm{CDCl}_{3}\right): \delta$ $27.8,29.2,44.8,66.0,71.6,73.3,81.5,127.7,128.5,128.7,135.6,136.8,168.3$. IR $\left(\mathrm{NaCl}, \mathrm{cm}^{-1}\right): v_{\mathrm{C}=\mathrm{O}}=1760 ; v_{\max }=2936,1497,1455 . \mathbf{M S}(70 \mathrm{eV}) \mathrm{m} / \mathrm{z}(\%): 344 / 6\left(\mathrm{M}^{+}+1\right.$, 100). Anal. Calcd for $\mathrm{C}_{20} \mathrm{H}_{22} \mathrm{ClNO}_{2}$ : C 69.86; H 6.45; N 4.07. Found: C 70.03; H 6.53; N 3.96.

\section{1-Benzyl-3-methoxy-4-(1-chloro-1-methylethyl)azetidin-2-one 1c}

Colourless oil, 75\% yield, TLC Rf 0.25 (petroleum ether/ethyl acetate 3/1). ${ }^{1} \mathbf{H}$ NMR $\left(300 \mathrm{MHz}, \mathrm{CDCl}_{3}\right): \delta 1.61$ and $1.67(6 \mathrm{H}, 2 \times \mathrm{s}), 3.52(3 \mathrm{H}, \mathrm{s}), 3.89(1 \mathrm{H}, \mathrm{d}, J=5.2 \mathrm{~Hz})$, 
$4.30(1 \mathrm{H}, \mathrm{d}, J=14.7 \mathrm{~Hz}), 4.38(1 \mathrm{H}, \mathrm{d}, J=5.2 \mathrm{~Hz}), 4.84(1 \mathrm{H}, \mathrm{d}, J=14.7 \mathrm{~Hz}) .{ }^{13} \mathbf{C}$ NMR (75 MHz, $\left.\mathrm{CDCl}_{3}\right): \delta 27.7,29.1,44.8,59.6,66.1,71.6,84.0,127.7,128.6,135.6,168.3$. IR $\left(\mathrm{NaCl}, \mathrm{cm}^{-1}\right): v_{\mathrm{C}=0}=1765 ; v_{\max }=2937,1496,1456 . \mathbf{M S}(70 \mathrm{eV}) \mathrm{m} / \mathrm{z}(\%): 268 / 70$ $\left(\mathrm{M}^{+}+1,100\right)$. Anal. Calcd for $\mathrm{C}_{14} \mathrm{H}_{18} \mathrm{ClNO}_{2}: \mathrm{C} 62.80 ; \mathrm{H}$ 6.78; $\mathrm{N}$ 5.23. Found: $\mathrm{C} 62.94 ; \mathrm{H}$ $6.71 ;$ N 5.35.

\section{3-Benzyloxy-1-tert-butyl-4-(1-chloro-1-methylethyl)azetidin-2-one $1 \mathrm{~g}$}

Colourless oil, 79\% yield, TLC Rf 0.35 (petroleum ether/ethyl acetate 3/1). ${ }^{1} \mathbf{H}$ NMR (300 MHz, $\left.\mathrm{CDCl}_{3}\right): \delta 1.48(9 \mathrm{H}, \mathrm{s}), 1.74$ and $1.75(6 \mathrm{H}, 2 \times \mathrm{s}), 4.14(1 \mathrm{H}, \mathrm{d}, J=5.8 \mathrm{~Hz})$, $4.52(1 \mathrm{H}, \mathrm{d}, J=5.8 \mathrm{~Hz}), 4.68(1 \mathrm{H}, \mathrm{d}, J=11.8 \mathrm{~Hz}), 4.90(1 \mathrm{H}, \mathrm{d}, J=11.8 \mathrm{~Hz}), 7.25-7.38$ $(5 \mathrm{H}, \mathrm{m}) .{ }^{13} \mathrm{C}$ NMR $\left(75 \mathrm{MHz}, \mathrm{CDCl}_{3}\right): \delta 27.3,29.8,28.8,54.8,71.5,68.3,73.2,79.4$, 127.7, 127.9, 128.5, 137.0, 168.7. IR $\left(\mathrm{NaCl}, \mathrm{cm}^{-1}\right): v_{\mathrm{C}=0}=1737 ; v_{\max }=2977,1645,1604$, 1497, 1455. MS $(70 \mathrm{eV}) \mathrm{m} / \mathrm{z}(\%): 310 / 2\left(\mathrm{M}^{+}+1,100\right)$. Anal. Calcd for $\mathrm{C}_{17} \mathrm{H}_{24} \mathrm{ClNO}_{2}: \mathrm{C}$ 65.90; H 7.81; N 4.52. Found: C 65.77; H 7.90; N 4.64.

\section{Cis-3-benzyloxy-4-[(2-bromo-1,1-dimethyl)ethyl]-1-t-butylazetidin-2-one 11a}

Colourless oil, 90\% yield, TLC Rf 0.25 (petroleum ether/ethyl acetate 6/1). ${ }^{1} \mathbf{H}$ NMR (300 MHz, $\left.\mathrm{CDCl}_{3}\right): \delta 1.20$ and $1.25(2 \times 3 \mathrm{H}, 2 \times \mathrm{s}), 1.38(9 \mathrm{H}, \mathrm{s}), 3.33(1 \mathrm{H}, \mathrm{d}, J=9.9$ $\mathrm{Hz}), 3.91(1 \mathrm{H}, \mathrm{d}, J=9.9 \mathrm{~Hz}), 3.96(1 \mathrm{H}, \mathrm{d}, J=5.8 \mathrm{~Hz}), 4.54(1 \mathrm{H}, \mathrm{d}, J=5.8 \mathrm{~Hz}), 4.71(1 \mathrm{H}$, d, $J=11.8 \mathrm{~Hz}), 4.93(1 \mathrm{H}, \mathrm{d}, J=11.8 \mathrm{~Hz}), 7.24-7.35(5 \mathrm{H}, \mathrm{m}) .{ }^{13} \mathbf{C}$ NMR $(75 \mathrm{MHz}$, $\left.\mathrm{CDCl}_{3}\right): \delta 22.6,26.5,28.9,37.2,44.5,53.9,65.1,73.2,79.7,127.6,127.8,128.4,137.2$ 169.5. IR $\left(\mathrm{NaCl}, \mathrm{cm}^{-1}\right): v_{\mathrm{C}=0}=1748 . \mathbf{M S}(70 \mathrm{eV}): \mathrm{m} / \mathrm{z}(\%) 370 / 368\left(\mathrm{M}^{+}+1,11\right) ; 362(26)$; 
361 (100). Anal. Calcd for $\mathrm{C}_{18} \mathrm{H}_{26} \mathrm{BrNO}_{2}$ : C 58.70; H 7.12; N 3.80. Found: C 58.84; H 7.19; N 3.65.

\section{Cis-1-benzyl-3-benzyloxy-4-[(2-bromo-1,1-dimethyl)ethyl]azetidin-2-one 11b}

Colourless oil, 69\% yield, TLC Rf 0.3 (petroleum ether/ethyl acetate 6/1). ${ }^{1}$ H NMR (300 $\left.\mathrm{MHz}, \mathrm{CDCl}_{3}\right): \delta 1.08$ and $1.12(2 \times 3 \mathrm{H}, 2 \times \mathrm{s}), 3.22(1 \mathrm{H}, \mathrm{d}, J=10.1 \mathrm{~Hz}), 3.62(1 \mathrm{H}, \mathrm{d}, J=$ $10.1 \mathrm{~Hz}), 3.71(1 \mathrm{H}, \mathrm{d}, J=5.4 \mathrm{~Hz}), 4.14(1 \mathrm{H}, \mathrm{d}, J=15.1 \mathrm{~Hz}), 4.68(1 \mathrm{H}, \mathrm{d}, J=5.4 \mathrm{~Hz})$, $4.70(1 \mathrm{H}, \mathrm{d}, J=11.8 \mathrm{~Hz}), 4.72(1 \mathrm{H}, \mathrm{d}, J=15.1 \mathrm{~Hz}), 4.95(1 \mathrm{H}, \mathrm{d}, J=11.8 \mathrm{~Hz}), 7.21-7.35$ $(10 \mathrm{H}, \mathrm{m}) .{ }^{13} \mathbf{C} \mathbf{N M R}\left(75 \mathrm{MHz}\right.$, ref $\left.=\mathrm{CDCl}_{3}\right): \delta 22.9,24.7,37.3,43.8,46.0,63.8,73.5$, $82.2,127.8,128.1,128.4,128.6,129.0,135.7,137.2,169.3 . \mathbf{I R}\left(\mathrm{NaCl}, \mathrm{cm}^{-1}\right): v_{\mathrm{C}=\mathrm{O}}=$ 1755. MS (70 eV): m/z (\%) 402/2 (M+1, 100); 403/405 (28); 350 (7); 307 (9); 282 (7); 254 (10). Anal. Calcd for $\mathrm{C}_{21} \mathrm{H}_{24} \mathrm{BrNO}_{2}$ : C 62.69; H 6.01; N 3.48. Found: $\mathrm{C} 62.54 ; \mathrm{H}$ $6.08 ;$ N 3.59.

\section{Cis-1-benzyl-4-[(2-bromo-1,1-dimethyl)ethyl]-3-methoxyazetidin-2-one 11c}

White crystals, 85\% yield, TLC Rf 0.3 (petroleum ether/ethyl acetate 6/1), Mp. $45^{\circ} \mathrm{C}{ }^{1} \mathbf{H}$ NMR (300 MHz, $\left.\mathrm{CDCl}_{3}\right): \delta 1.05$ and $1.08(2 \times 3 \mathrm{H}, 2 \times \mathrm{s}), 3.20(1 \mathrm{H}, \mathrm{d}, J=10.1 \mathrm{~Hz}), 3.56$ $(1 \mathrm{H}, \mathrm{d}, J=10.1 \mathrm{~Hz}), 3.56(3 \mathrm{H}, \mathrm{s}), 3.70(1 \mathrm{H}, \mathrm{d}, J=5.2 \mathrm{~Hz}), 4.16(1 \mathrm{H}, \mathrm{d}, J=15.1 \mathrm{~Hz})$, $4.49(1 \mathrm{H}, \mathrm{d}, J=5.2 \mathrm{~Hz}), 4.70(1 \mathrm{H}, \mathrm{d}, J=15.1 \mathrm{~Hz}), 7.22-7.35(5 \mathrm{H}, \mathrm{m}) .{ }^{13} \mathrm{C}$ NMR $(75$ $\left.\mathrm{MHz}, \mathrm{CDCl}_{3}\right): \delta 22.7,24.3,37.1,43.5,45.8,59.5,63.7,84.6,127.8,128.2,128.8,135.6$, 169.1. IR $\left(\mathrm{KBr}, \mathrm{cm}^{-1}\right): \mathrm{v}_{\mathrm{C}=0}=1756$. MS $(70 \mathrm{eV}): \mathrm{m} / \mathrm{z}(\%) 326 / 8\left(\mathrm{M}^{+}+1,100\right)$. Anal. Calcd for $\mathrm{C}_{15} \mathrm{H}_{20} \mathrm{BrNO}_{2}$ : C 55.23; H 6.18; N 4.29. Found: C 55.31; H 6.02; N 4.38. 
General procedure for the synthesis of aziridines 2 and azetidines 12 from 4(haloalkyl)azetidin-2-ones 1 and 11

As an example, the synthesis of 2-benzyloxy-2-(1-isopropyl-3,3-dimethylaziridin-2yl)ethanol 2a is described. To a mixture of diethyl ether $(50 \mathrm{ml})$ and $\mathrm{LiAlH}_{4}(0.72 \mathrm{~g}, 19.0$ mmol) at $0^{\circ} \mathrm{C}$, a solution of 3-benzyloxy-4-(1-chloro-1-methylethyl)-1-isopropylazetidin2-one 1a $(1 \mathrm{~g}, 3.8 \mathrm{mmol})$ in diethyl ether $(20 \mathrm{ml})$ is added dropwise over a period of 5 minutes. After two hours of stirring at $0^{\circ} \mathrm{C}$, little water is added slowly in order to neutralise the residual hydride. The solvent is decanted and the residue is washed three times with $20 \mathrm{ml}$ of diethyl ether. After drying $\left(\mathrm{MgSO}_{4}\right)$ of the combined organic fractions and filtration, the solvent is evaporated under reduced pressure. The crude reaction mixture is purified by flash chromatography $\left(\mathrm{CH}_{2} \mathrm{Cl}_{2} / \mathrm{MeOH} 20 / 1\right.$ ratio on silicagel), affording 2-benzyloxy-2-(1-isopropyl-3,3-dimethylaziridin-2-yl)ethanol 2a in 57\% yield and 3-benzyloxy-2-(1-chloro-1-methylethyl)-1-isopropylazetidine 3a in 3\% yield.

\section{2-Benzyloxy-2-(1-isopropyl-3,3-dimethylaziridin-2-yl)ethanol 2a}

Colourless oil, 57\% yield, TLC Rf $0.3\left(\mathrm{CH}_{2} \mathrm{Cl}_{2} / \mathrm{MeOH} 20 / 1\right) .{ }^{1} \mathbf{H}$ NMR $(270 \mathrm{MHz}$, $\left.\mathrm{CDCl}_{3}\right): \delta 1.10$ and $1.22(6 \mathrm{H}, 2 \times \mathrm{s}), 1.19$ and $1.24(6 \mathrm{H}, 2 \times \mathrm{s}), 1.40(1 \mathrm{H}, \mathrm{d}, J=8.6 \mathrm{~Hz})$, $2.20(1 \mathrm{H}$, septet, $J=6 \mathrm{~Hz}), 2.40(1 \mathrm{H}$, broad s), 3.29-3.35 $(1 \mathrm{H}, \mathrm{m}), 3.50-3.66(2 \mathrm{H}, \mathrm{m})$, $4.61(1 \mathrm{H}, \mathrm{d}, J=11 \mathrm{~Hz}), 4.89(1 \mathrm{H}, \mathrm{d}, J=11 \mathrm{~Hz}), 7.24-7.39(5 \mathrm{H}, \mathrm{m}) .{ }^{13} \mathbf{C} \mathbf{N M R}(67.5$ $\left.\mathrm{MHz}, \mathrm{CDCl}_{3}\right): \delta 18.3,23.0,23.1,23.3,39.0,50.0,53.1,63.5,72.9,80.8,127.6,127.7$, 
128.4, 138.7. IR $\left(\mathrm{NaCl}, \mathrm{cm}^{-1}\right): v_{\max }=2967,1497,1454,1382 . \mathbf{M S}(70 \mathrm{eV}) \mathrm{m} / \mathrm{z}(\%): 264$ $\left(\mathrm{M}^{+}+1,100\right)$. Anal. Calcd for $\mathrm{C}_{16} \mathrm{H}_{25} \mathrm{NO}_{2}$ : $\mathrm{C} 72.96 ; \mathrm{H}$ 9.57; N 5.32. Found: $\mathrm{C} 72.76 ; \mathrm{H}$ 9.70; N 5.44.

\section{2-(1-Benzyl-3,3-dimethylaziridin-2-yl)-2-benzyloxyethanol 2b}

Colourless oil, $62 \%$ yield, TLC Rf $0.2\left(\mathrm{CH}_{2} \mathrm{Cl}_{2} / \mathrm{MeOH} 20 / 1\right) .{ }^{1} \mathbf{H}$ NMR $(300 \mathrm{MHz}$, $\left.\mathrm{CDCl}_{3}\right): \delta 1.21$ and $1.22(6 \mathrm{H}, 21.53(1 \mathrm{H}, \mathrm{d}, J=8.5 \mathrm{~Hz}), 2.43(1 \mathrm{H}$, broad s), 3.30-3.37 $(1 \mathrm{H}, \mathrm{m}), 3.53-3.59(3 \mathrm{H}, \mathrm{m}), 3.63(1 \mathrm{H}, \mathrm{d}, J=14.2 \mathrm{~Hz}), 3.75(1 \mathrm{H}, \mathrm{d}, J=14.2 \mathrm{~Hz}), 4.41$ $(1 \mathrm{H}, \mathrm{d}, J=11.7 \mathrm{~Hz}), 4.53(1 \mathrm{H}, \mathrm{d}, J=11.7 \mathrm{~Hz}), 7.20-7.40(10 \mathrm{H}, \mathrm{m}) .{ }^{13} \mathbf{C} \mathbf{~ N M R}(75 \mathrm{MHz}$, $\left.\mathrm{CDCl}_{3}\right): \delta 18.4,23.0,38.6,50.7,56.8,63.7,71.6,80.4,126.8,127.5,127.7,128.0,128.3$, 128.4, 138.6, 140.1. IR $\left(\mathrm{NaCl}, \mathrm{cm}^{-1}\right): v_{\max }=3401,2925,2871,1606,1496,1454,1378$. MS $(70 \mathrm{eV}) \mathrm{m} / \mathrm{z}(\%): 312\left(\mathrm{M}^{+}+1,100\right)$. Anal. Calcd for $\mathrm{C}_{20} \mathrm{H}_{25} \mathrm{NO}_{2}: \mathrm{C} 77.14 ; \mathrm{H}$ 8.09; N 4.50. Found: C 77.02; H 7.92; N 4.64.

\section{2-(1-Benzyl-3,3-dimethylaziridin-2-yl)-2-methoxyethanol 2c}

Colourless oil, 55\% yield, TLC Rf $0.15\left(\mathrm{CH}_{2} \mathrm{Cl}_{2} / \mathrm{MeOH}\right.$ 20/1). ${ }^{1} \mathbf{H}$ NMR (300 MHz, $\left.\mathrm{CDCl}_{3}\right): \delta 1.21(6 \mathrm{H}, 2 \times \mathrm{s}), 1.43(1 \mathrm{H}, \mathrm{d}, J=8.5 \mathrm{~Hz}), 2.75(1 \mathrm{H}$, broad s), 3.08-3.14 $(1 \mathrm{H}, \mathrm{m})$, $3.32(3 \mathrm{H}, \mathrm{s}), 3.49-3.62(3 \mathrm{H}, \mathrm{m}), 3.85$ (1H, d, $J=14.3 \mathrm{~Hz}), 7.20-7.38(5 \mathrm{H}, \mathrm{m}) .{ }^{13} \mathbf{C}$ NMR (75 MHz, $\left.\mathrm{CDCl}_{3}\right): \delta 18.4,23.0,38.5,50.5,56.7,57.7,63.5,82.2,126.7,127.8,128.3$, 140.1. IR $\left(\mathrm{NaCl}, \mathrm{cm}^{-1}\right): v_{\max }=3411,2983,2929,2828,1606,1496,1453,1422,1378$. MS $(70 \mathrm{eV}) \mathrm{m} / \mathrm{z}(\%): 236\left(\mathrm{M}^{+}+1,100\right)$. Anal. Calcd for $\mathrm{C}_{14} \mathrm{H}_{21} \mathrm{NO}_{2}: \mathrm{C} 71.46 ; \mathrm{H}$ 8.99; $\mathrm{N}$ 5.95. Found: C 71.32; H 8.90; N 6.14. 


\section{2-(1-Allyl-3,3-dimethylaziridin-2-yl)-2-benzyloxyethanol 2d}

Colourless oil, 43\% yield, TLC Rf $0.2\left(\mathrm{CH}_{2} \mathrm{Cl}_{2} / \mathrm{MeOH} 20 / 1\right) .{ }^{1} \mathbf{H}$ NMR (300 MHz, $\left.\mathrm{CDCl}_{3}\right): \delta 1.20$ and $1.21(6 \mathrm{H}, 2 \times \mathrm{s}), 1.40(1 \mathrm{H}, \mathrm{d}, J=8.5 \mathrm{~Hz}), 2.10(1 \mathrm{H}$, broad s $), 3.08(1 \mathrm{H}$, $\mathrm{d} \times \mathrm{d}, J=14.6 \mathrm{~Hz}, J=5.1 \mathrm{~Hz}), 3.26(1 \mathrm{H}, \mathrm{d} \times \mathrm{d}, J=14.6 \mathrm{~Hz}, J=6.1 \mathrm{~Hz}), 3.33-3.40(1 \mathrm{H}, \mathrm{m})$, 3.59-3.61 $(2 \mathrm{H}, \mathrm{m}), 4.60(1 \mathrm{H}, \mathrm{d}, J=11.3 \mathrm{~Hz}), 4.91(1 \mathrm{H}, \mathrm{d}, J=11.3 \mathrm{~Hz}), 5.11-5.29(2 \mathrm{H}$, m), 5.89-6.08 (1H, m), 7.28-7.37 (5H, m). ${ }^{13} \mathbf{C}$ NMR $\left(75 \mathrm{MHz}, \mathrm{CDCl}_{3}\right): \delta$ 18.1, 23.0, $38.3,50.5,55.7,63.7,71.9,80.7,116.1,127.6,127.8,128.4,136.4,138.6 . \mathbf{I R}\left(\mathrm{NaCl}, \mathrm{cm}^{-}\right.$ $\left.{ }^{1}\right): v_{\max }=3409,3088,3065,2984,2924,2871,1644,1497,1455,1418,1377 . \mathbf{M S}(70 \mathrm{eV})$ m/z (\%): $262\left(\mathrm{M}^{+}+1,100\right) ;$ 91(12). Anal. Calcd for $\mathrm{C}_{16} \mathrm{H}_{23} \mathrm{NO}_{2}$ : C 73.53; H 8.87; N 5.36. Found: C 73.41; H 8.99; N 5.44.

\section{2-(1-Allyl-3,3-dimethylaziridin-2-yl)-2-methoxyethanol 2e}

Colourless oil, 51\% yield, TLC Rf $0.12\left(\mathrm{CH}_{2} \mathrm{Cl}_{2} / \mathrm{MeOH} 20 / 1\right) .{ }^{1} \mathbf{H}$ NMR (300 MHz, $\left.\mathrm{CDCl}_{3}\right): \delta 1.17$ and $1.19(6 \mathrm{H}, 2 \times \mathrm{s}), 1.29(1 \mathrm{H}, \mathrm{d}, J=8.5 \mathrm{~Hz}), 2.32(1 \mathrm{H}$, broad s), $3.02(1 \mathrm{H}$, $\mathrm{d} \times \mathrm{d}, J=14.9 \mathrm{~Hz}, J=5.5 \mathrm{~Hz}), 3.08-3.18(1 \mathrm{H}, \mathrm{m}), 3.32(1 \mathrm{H}, \mathrm{d} \times \mathrm{d}, J=14.9 \mathrm{~Hz}, J=5.8$ $\mathrm{Hz}), 3.47-3.64(2 \mathrm{H}, \mathrm{m}), 3.53(3 \mathrm{H}, \mathrm{s}), 5.10-5.27(2 \mathrm{H}, \mathrm{m}), 5.92-6.05(1 \mathrm{H}, \mathrm{m}) .{ }^{13} \mathrm{C}$ NMR $\left(75 \mathrm{MHz}_{\mathrm{CDCl}}\right): \delta 18.1,22.9,38.350 .5,55.6,57.7,63.2,82.3,115.8,136.4 . \mathbf{I R}(\mathrm{NaCl}$, $\left.\mathrm{cm}^{-1}\right): v_{\max }=3401,2984,2928,1644,1463,1418,1378 . \mathbf{M S}(70 \mathrm{eV}) \mathrm{m} / \mathrm{z}(\%): 186$ $\left(\mathrm{M}^{+}+1,100\right) ; 112$ (50). Anal. Calcd for $\mathrm{C}_{10} \mathrm{H}_{19} \mathrm{NO}_{2}$ : C 64.83; H 10.34; N 7.56. Found: C 64.98; H 10.49; N 7.40.

\section{2-(1-Cyclohexyl-3,3-dimethylaziridin-2-yl)-2-methoxyethanol $2 f$}


Colourless oil, 52\% yield, TLC Rf $0.1\left(\mathrm{CH}_{2} \mathrm{Cl}_{2} / \mathrm{MeOH} 20 / 1\right) .{ }^{1} \mathbf{H}$ NMR $(270 \mathrm{MHz}$, $\left.\mathrm{CDCl}_{3}\right): \delta 1.17$ and $1.24(6 \mathrm{H}, 2 \times \mathrm{s}), 1.21-1.94(11 \mathrm{H}, \mathrm{m}), 1.31(1 \mathrm{H}, \mathrm{d}, J=8.6 \mathrm{~Hz}), 2.50$ $(1 \mathrm{H}$, broad s), 3.02-3.09 $(1 \mathrm{H}, \mathrm{m}), 3.51(1 \mathrm{H}, \mathrm{d} \times \mathrm{d}, J=12 \mathrm{~Hz}, J=7 \mathrm{~Hz}), 3.54(3 \mathrm{H}, \mathrm{s}), 3.62$ $(1 \mathrm{H}, \mathrm{d} \times \mathrm{d}, J=12 \mathrm{~Hz}, J=4 \mathrm{~Hz}) .{ }^{13} \mathbf{C} \mathbf{N M R}\left(67.5 \mathrm{MHz}, \mathrm{CDCl}_{3}\right): \delta 18.3,23.4,24.8,25.2$, $25.9,32.9,33.8,38.2,49.2,58.8,61.4,63.2,82.2 . \mathbf{I R}\left(\mathrm{NaCl}, \mathrm{cm}^{-1}\right): v_{\max }=2982,1451$, 1379, 1364, 1348. MS (70 eV) m/z (\%): $228\left(\mathrm{M}^{+}+1,100\right)$. Anal. Calcd for $\mathrm{C}_{13} \mathrm{H}_{25} \mathrm{NO}_{2}: \mathrm{C}$ 68.68; H 11.08; N 6.16. Found: C 68.89; H 11.01; N 6.27.

\section{3-Benzyloxy-2-(1-chloro-1-methylethyl)-1-isopropylazetidine 3a}

Colourless oil, 3\% yield, TLC Rf 0.25 (petroleum ether/ethyl acetate 20/1). ${ }^{1} \mathbf{H}$ NMR (300 MHz, $\left.\mathrm{CDCl}_{3}\right): \delta 0.97$ and $1.03(6 \mathrm{H}, 2 \times \mathrm{d}, J=6.6 \mathrm{~Hz}), 1.71$ and $1.82(6 \mathrm{H}, 2 \times \mathrm{s}), 3.09$ $(1 \mathrm{H}$, septet, $J=6.6 \mathrm{~Hz}), 3.17(1 \mathrm{H}, \mathrm{d} \times \mathrm{d}, J=8.7 \mathrm{~Hz}, J=6.3 \mathrm{~Hz}), 3.21(1 \mathrm{H}, \mathrm{d} \times \mathrm{d} \times \mathrm{d}, J=8.7$ $\mathrm{Hz}, J=3.6 \mathrm{~Hz}, J=1.1 \mathrm{~Hz}), 3.71(1 \mathrm{H}, \mathrm{d} \times \mathrm{d}, J=7.2 \mathrm{~Hz}, J=1.1 \mathrm{~Hz}), 4.19(1 \mathrm{H}, \mathrm{d} \times \mathrm{d} \times \mathrm{d}, J=$ $7.2 \mathrm{~Hz}, J=6.3 \mathrm{~Hz}, J=3.6 \mathrm{~Hz}), 4.36(1 \mathrm{H}, \mathrm{d}, J=12.1 \mathrm{~Hz}), 4.52(1 \mathrm{H}, \mathrm{d}, J=12.1 \mathrm{~Hz})$, 7.24-7.36 (5H, m). $\left.{ }^{13} \mathbf{C ~ N M R ~ ( 7 5 ~ M H z , ~} \mathrm{CDCl}_{3}\right): \delta 14.7,20.9,29.3,29.5,49.9,50.6,71.1$, 72.1, 72.8, 73.0, 127.5, 127.6, 128.3, 138.1. IR $\left(\mathrm{NaCl}, \mathrm{cm}^{-1}\right): v_{\max }=2965,2932,2868$, 1497, 1455. MS $(70 \mathrm{eV}) \mathrm{m} / \mathrm{z}(\%): 282 / 4\left(\mathrm{M}^{+}+1,100\right)$. Anal. Calcd for $\mathrm{C}_{16} \mathrm{H}_{24} \mathrm{ClNO}$ : C 68.19; H 8.58; N 4.97. Found: C 68.30; H 8.69; N 5.13.

\section{1-Benzyl-3-benzyloxy-2-(1-chloro-1-methylethyl)azetidine 3b}

Colourless oil, 4\% yield, TLC Rf 0.2 (petroleum ether/ethyl acetate 9/1). ${ }^{1} \mathbf{H}$ NMR (300 $\left.\mathrm{MHz}, \mathrm{CDCl}_{3}\right): \delta 1.73$ and $1.93(6 \mathrm{H}, 2 \times \mathrm{s}), 2.86(1 \mathrm{H}, \mathrm{d} \times \mathrm{d}, J=8.8 \mathrm{~Hz}, J=6.1 \mathrm{~Hz}),$, $(1 \mathrm{H}, \mathrm{d}, J=8.8 \mathrm{~Hz}), 3.45(1 \mathrm{H}, \mathrm{d}, J=13.2 \mathrm{~Hz}), 3.66(1 \mathrm{H}, \mathrm{d}, J=7.2 \mathrm{~Hz}), 4.23-4.28(1 \mathrm{H}$, 
m), $4.29(1 \mathrm{H}, \mathrm{d}, J=12.3 \mathrm{~Hz}), 4.36(1 \mathrm{H}, \mathrm{d}, J=13.2 \mathrm{~Hz}), 4.48(1 \mathrm{H}, \mathrm{d}, J=12.3 \mathrm{~Hz}), 7.21-$ $7.36(5 \mathrm{H}, \mathrm{m}) .{ }^{13} \mathrm{C}$ NMR $\left(75 \mathrm{MHz}, \mathrm{CDCl}_{3}\right): \delta 29.3,29.6,56.6,62.1,70.9,72.5,73.2$, 76.7, 126.9, 127.4, 127.6, 128.2, 128.3, 128.7, 137.9, 138.4. IR $\left(\mathrm{NaCl}, \mathrm{cm}^{-1}\right): v_{\max }=3029$, 2975, 2932, 2863, 1495, 1454, 1368. MS (70 eV) m/z (\%): 332/0 ( $\left.\mathbf{M}^{+}+1,100\right)$. Anal. Calcd for $\mathrm{C}_{20} \mathrm{H}_{24} \mathrm{ClNO}$ : C 72.82; H 7.33; N 4.25. Found: C 72.68; H 7.44; N 4.32.

\section{1-Benzyl-2-(1-chloro-1-methylethyl)-3-methoxyazetidine 3c}

Colourless oil, 4\% yield, TLC Rf 0.2 (petroleum ether/ethyl acetate 9/1). ${ }^{1} \mathbf{H}$ NMR (300 $\left.\mathrm{MHz}, \mathrm{CDCl}_{3}\right): \delta 1.69$ and $1.88(6 \mathrm{H}, 2 \times \mathrm{s}), 2.81(1 \mathrm{H}, \mathrm{d} \times \mathrm{d}, J=8.9 \mathrm{~Hz}, J=5.9 \mathrm{~Hz}), 3.18$ $(3 \mathrm{H}, \mathrm{s}), 3.29(1 \mathrm{H}, \mathrm{d}, J=8.9 \mathrm{~Hz}), 3.42(1 \mathrm{H}, \mathrm{d}, J=13.4 \mathrm{~Hz}), 3.61(1 \mathrm{H}, \mathrm{d}, J=6.9 \mathrm{~Hz})$, 4.01-4.06 (1H, m), $4.36(1 \mathrm{H}, \mathrm{d}, J=13.4 \mathrm{~Hz}), 7.21-7.36(5 \mathrm{H}, \mathrm{m}) .{ }^{13} \mathbf{C} \mathbf{~ N M R}(75 \mathrm{MHz}$, $\left.\mathrm{CDCl}_{3}\right): \delta 29.2,29.5,56.2,56.4,62.2,73.0,74.8,76.8,126.9,128.2,128.7,138.3$. IR $\left(\mathrm{NaCl}, \mathrm{cm}^{-1}\right): v_{\max }=2978,2932,2833,1495,1453,1368 . \mathbf{M S}(70 \mathrm{eV}) \mathrm{m} / \mathrm{z}(\%): 254 / 6$ $\left(\mathrm{M}^{+}+1,100\right)$. Anal. Calcd for $\mathrm{C}_{14} \mathrm{H}_{20} \mathrm{ClNO}$ : $\mathrm{C} 66.26 ; \mathrm{H}$ 7.94; N 5.52. Found: $\mathrm{C} 66.38 ; \mathrm{H}$ 7.80; N 5.71.

\section{1-Allyl-3-benzyloxy-2-(1-chloro-1-methylethyl)azetidine 3d}

Colourless oil, 2\% yield, TLC Rf 0.3 (petroleum ether/ethyl acetate 9/1). ${ }^{1} \mathbf{H}$ NMR (300 $\left.\mathrm{MHz}, \mathrm{CDCl}_{3}\right): \delta 1.69$ and $1.85(6 \mathrm{H}, 2 \times \mathrm{s}), 2.89(1 \mathrm{H}, \mathrm{d} \times \mathrm{d}, J=9.1 \mathrm{~Hz}, J=5.9 \mathrm{~Hz}), 2.94$ $(1 \mathrm{H}, \mathrm{d} \times \mathrm{d}, J=13.5 \mathrm{~Hz}, J=7.4 \mathrm{~Hz}), 3.42(1 \mathrm{H}, \mathrm{d} \times \mathrm{d} \times \mathrm{d}, J=9.1 \mathrm{~Hz}, J=2.2 \mathrm{~Hz}, J=1.4 \mathrm{~Hz})$, $3.52(1 \mathrm{H}, \mathrm{d} \times \mathrm{d}, J=6.9 \mathrm{~Hz}, J=1.0 \mathrm{~Hz}), 3.72(1 \mathrm{H}, \mathrm{d} \times \mathrm{d} \times \mathrm{t}, J=13.8 \mathrm{~Hz}, J=5.0 \mathrm{~Hz}, J=1.7$ $\mathrm{Hz}), 4.25(1 \mathrm{H}, \mathrm{d} \times \mathrm{d} \times \mathrm{d}, J=6.9 \mathrm{~Hz}, J=6.1 \mathrm{~Hz}, J=2.5 \mathrm{~Hz}), 4.32(1 \mathrm{H}, \mathrm{d}, J=12.0 \mathrm{~Hz}), 4.52$ $(1 \mathrm{H}, \mathrm{d}, J=12.0 \mathrm{~Hz}), 5.08-5.22(2 \mathrm{H}, \mathrm{m}), 5.78-5.91(1 \mathrm{H}, \mathrm{m}), 7.27-7.37(5 \mathrm{H}, \mathrm{m}) .{ }^{13} \mathbf{C}$ NMR 
$\left(67.8 \mathrm{MHz}, \mathrm{CDCl}_{3}\right): \delta 29.2,29.5,56.3,60.8,70.9,72.5,72.7,76.7,117.1,127.3,128.3$,

127.5, 134.5, 137.8. IR $\left(\mathrm{NaCl}, \mathrm{cm}^{-1}\right): v_{\max }=3067,3031,2978,2933,2861,2841,2794$, 1643, 1497, 1455, 1383, 1365, 1355. MS (70 eV) m/z (\%): 280/2 ( $\left.\mathrm{M}^{+}+1,100\right)$. Anal. Calcd for $\mathrm{C}_{16} \mathrm{H}_{22} \mathrm{ClNO}$ : C 68.68; H 7.93; N 5.01. Found: C 68.60; H 8.03; N 5.05.

\section{1-Allyl-2-(1-chloro-1-methylethyl)-3-methoxyazetidine 3e}

Colourless oil, 3\% yield, TLC Rf 0.3 (petroleum ether/ethyl acetate 9/1). ${ }^{1} \mathbf{H}$ NMR (300 $\left.\mathrm{MHz}, \mathrm{CDCl}_{3}\right): \delta 1.64$ and $1.80(6 \mathrm{H}, 2 \times \mathrm{s}), 2.89(1 \mathrm{H}, \mathrm{d} \times \mathrm{d}, J=8.9 \mathrm{~Hz}, J=5.8 \mathrm{~Hz}), 2.92$ $(1 \mathrm{H}, \mathrm{d} \times \mathrm{d}, J=13.5 \mathrm{~Hz}, J=7.4 \mathrm{~Hz}), 3.22(3 \mathrm{H}, \mathrm{s}), 3.37(1 \mathrm{H}, \mathrm{d} \times \mathrm{d} \times \mathrm{d}, J=8.8 \mathrm{~Hz}, J=1.9 \mathrm{~Hz}$, $J=1.4 \mathrm{~Hz}), 3.49(1 \mathrm{H}, \mathrm{d}, J=6.6 \mathrm{~Hz}), 3.74(1 \mathrm{H}, \mathrm{d} \times \mathrm{d} \times \mathrm{t}, J=13.8 \mathrm{~Hz}, J=5.0 \mathrm{~Hz}, J=1.5$ $\mathrm{Hz}), 4.03(1 \mathrm{H}, \mathrm{d} \times \mathrm{d} \times \mathrm{d}, J=6.7 \mathrm{~Hz}, J=5.8 \mathrm{~Hz}, J=2.1 \mathrm{~Hz}), 5.08-5.23(2 \mathrm{H}, \mathrm{m}), 5.78-5.91$ $(1 \mathrm{H}, \mathrm{m}) .{ }^{13} \mathbf{C} \mathbf{N M R}\left(67.8 \mathrm{MHz}, \mathrm{CDCl}_{3}\right): \delta 29.2,29.4,55.9,56.4,60.8,72.5,74.8,76.8$, 117.3, 134.3. IR $\left(\mathrm{NaCl}, \mathrm{cm}^{-1}\right): v_{\max }=3079,2982,2934,2834,1644,1461,1383,1367$, 1193. MS (70 eV) m/z (\%): 204/6 ( $\left.\mathrm{M}^{+}+1,100\right)$. Anal. Calcd for $\mathrm{C}_{10} \mathrm{H}_{18} \mathrm{ClNO}$ : $\mathrm{C} 58.96 ; \mathrm{H}$ 8.91; N 6.88. Found: C 59.09; H 9.02; N 6.69.

\section{2-(1-Chloro-1-methylethyl)-1-cyclohexyl-3-methoxyazetidine 3f}

Colourless oil, 5\% yield, TLC Rf 0.15 (petroleum ether/ethyl acetate 20/1). ${ }^{1} \mathbf{H}$ NMR (300 MHz, $\left.\mathrm{CDCl}_{3}\right): \delta 0.83-1.95(10 \mathrm{H}, \mathrm{m}), 1.66$ and $1.77(6 \mathrm{H}, 2 \times \mathrm{s}), 3.18(2 \mathrm{H}, \mathrm{d}, J=4.7$ Hz), $3.23(3 \mathrm{H}, \mathrm{s}), 3.77(1 \mathrm{H}, \mathrm{d}, 6.9 \mathrm{~Hz}), 3.96(1 \mathrm{H}, \mathrm{d} \times \mathrm{t}, J=6.9 \mathrm{~Hz}, J=4.4 \mathrm{~Hz}) .{ }^{13} \mathbf{C}$ NMR (75 MHz, $\left.\mathrm{CDCl}_{3}\right): \delta 25.3,25.6,25.8,26.3,31.4,29.3,29.5,50.7,56.6,72.6,72.9,74.5$. IR $\left(\mathrm{NaCl}, \mathrm{cm}^{-1}\right): v_{\max }=2929,2854,1450 . \mathbf{M S}(70 \mathrm{eV}) \mathrm{m} / \mathrm{z}(\%): 246 / 8\left(\mathrm{M}^{+}+1,100\right)$. Anal. Calcd for $\mathrm{C}_{13} \mathrm{H}_{24} \mathrm{ClNO}$ : C 63.53; H 9.84; N 5.70. Found: C 63.41; H 9.95; N 5.66. 


\section{3-Benzyloxy-1-tert-butyl-2-(1-chloro-1-methylethyl)azetidine 3g}

Colourless oil, $1 \%$ yield, TLC Rf 0.25 (petroleum ether/ethyl acetate 9/1). ${ }^{1}$ H NMR (300 $\left.\mathrm{MHz}, \mathrm{CDCl}_{3}\right): \delta 1.06(9 \mathrm{H}, \mathrm{s}), 1.77$ and $1.80(6 \mathrm{H}, 2 \times \mathrm{s}), 3.19(1 \mathrm{H}, \mathrm{d} \times \mathrm{d}, J=8.9 \mathrm{~Hz}, J=7.0$ $\mathrm{Hz}), 3.29(1 \mathrm{H}, \mathrm{d} \times \mathrm{d} \times \mathrm{d}, J=8.9 \mathrm{~Hz}, J=4.7 \mathrm{~Hz}, J=1.0 \mathrm{~Hz}), 3.71(1 \mathrm{H}, \mathrm{d} \times \mathrm{d}, J=7.4 \mathrm{~Hz}, J=$ $1.0 \mathrm{~Hz}), 4.14(1 \mathrm{H}, \mathrm{d} \times \mathrm{t}, J=7.2 \mathrm{~Hz}, J=4.7 \mathrm{~Hz}), 4.40(1 \mathrm{H}, \mathrm{d}, J=11.8 \mathrm{~Hz}), 4.5(1 \mathrm{H}, \mathrm{d}, J=$ $11.8 \mathrm{~Hz}), 7.22-7.35(5 \mathrm{H}, \mathrm{m}) .{ }^{13} \mathbf{C}$ NMR $\left(75 \mathrm{MHz}, \mathrm{CDCl}_{3}\right): \delta 26.1,29.6,51.6,52.3,75.5$, 71.1, 71.5, 72.0, 127.5, 128.1, 138.1. IR $\left(\mathrm{NaCl}, \mathrm{cm}^{-1}\right): v_{\max }=2970,2936,2871,1497$, 1455, 1364. MS $(70 \mathrm{eV}) \mathrm{m} / \mathrm{z}(\%): 296 / 8\left(\mathrm{M}^{+}+1,100\right)$. Anal. Calcd for $\mathrm{C}_{17} \mathrm{H}_{26} \mathrm{ClNO}_{2}: \mathrm{C}$ 69.02; H 8.86; N 4.73. Found: C 69.13; H 8.79; N 4.85.

\section{2-Benzyloxy-2-(1-t-butyl-3,3-dimethylazetidin-2-yl)ethanol 12a}

Colourless oil, 45\% yield, TLC Rf $0.2\left(\mathrm{CH}_{2} \mathrm{Cl}_{2} / \mathrm{MeOH} 33 / 1\right) .{ }^{1} \mathbf{H}$ NMR (300 MHz, $\left.\mathrm{CDCl}_{3}\right): \delta 0.95(9 \mathrm{H}, \mathrm{s}), 1.10$ and $1.36(6 \mathrm{H}, 2 \times \mathrm{s}), 2.83(1 \mathrm{H}, \mathrm{d}, J=7.3 \mathrm{~Hz}), 2.86(1 \mathrm{H}, \mathrm{d}, J=$ $7.3 \mathrm{~Hz}), 3.44(1 \mathrm{H}, \mathrm{d}, J=6.6 \mathrm{~Hz}), 3.66-3.78(2 \mathrm{H}, \mathrm{m}), 4.08(1 \mathrm{H}, \mathrm{d} \times \mathrm{d}, J=10.5 \mathrm{~Hz}, J=6.6$ $\mathrm{Hz}), 4.53(1 \mathrm{H}, \mathrm{d}, J=11.7 \mathrm{~Hz}), 4.58(1 \mathrm{H}, \mathrm{d}, J=11.7 \mathrm{~Hz}), 7.19-7.39(5 \mathrm{H}, \mathrm{m}) .{ }^{13} \mathbf{C}$ NMR $\left(75 \mathrm{MHz}\right.$, ref $\left.=\mathrm{CDCl}_{3}\right): \delta 24.3,29.5,25.5,32.7,52.5,57.8,63.3,67.1,72.3,78.3,126.9$, 127.7, 127.8, 128.4, 138.8. IR $\left(\mathrm{NaCl}, \mathrm{cm}^{-1}\right): v_{\mathrm{OH}}=3401 ; v_{\max }=3089,3065,3032,2965$, 2871, 1497, 1467, 1455, 1389, 1365, 1242, 1215, 1076, 1028. MS (70 eV): m/z (\%) 292 $\left(\mathrm{M}^{+}+1,100\right)$. Anal. Calcd for $\mathrm{C}_{18} \mathrm{H}_{29} \mathrm{NO}_{2}$ : C 74.18; $\mathrm{H} 10.06 ; \mathrm{N}$ 4.81. Found: $\mathrm{C} 74.10 ; \mathrm{H}$ $9.90 ; \mathrm{N} 4.68$. 


\section{2-(1-Benzyl-3,3-dimethylazetidin-2-yl)-2-benzyloxyethanol 12b}

Colourless oil, 52\% yield TLC Rf $0.2\left(\mathrm{CH}_{2} \mathrm{Cl}_{2} / \mathrm{MeOH} 33 / 1\right) .{ }^{1} \mathbf{H}$ NMR (300 MHz, $\left.\mathrm{CDCl}_{3}\right): \delta 1.16$ and $1.34(6 \mathrm{H}, 2 \times \mathrm{s}), 2.63(1 \mathrm{H}, \mathrm{d}, J=6.6 \mathrm{~Hz}), 3.09(1 \mathrm{H}, \mathrm{d}, J=6.6 \mathrm{~Hz})$, $3.16(1 \mathrm{H}, \mathrm{d}, J=9.6 \mathrm{~Hz}), 3.46(1 \mathrm{H}, \mathrm{d}, J=13.2 \mathrm{~Hz}), 3.48-3.56(1 \mathrm{H}, \mathrm{m}), 3.71-3.81(1 \mathrm{H}, \mathrm{m})$, 3.90-3.98 (1H, m), $4.35(1 \mathrm{H}, \mathrm{d}, J=13.2 \mathrm{~Hz}), 4.86(1 \mathrm{H}, \mathrm{d}, J=11.3 \mathrm{~Hz}), 4.92(1 \mathrm{H}, \mathrm{d}, J=$ 11.3 Hz), 7.28-7.51 (10H, m). ${ }^{13} \mathbf{C}$ NMR (75 MHz, ref $\left.=\mathrm{CDCl}_{3}\right): \delta 23.0,28.5,34.1,62.2$, $63.7,65.1,73.2,73.3,82.0,127.8,128.1,128.5,128.6,128.7,128.8,129.1,138.6,139.1$. IR $\left(\mathrm{NaCl}, \mathrm{cm}^{-1}\right): v_{\mathrm{OH}}=3393 ; v_{\max }=3087,3063,3029,2952,2926,2868,1496,1464$, 1454, 1368, 1359, 1097, 1068, 1028. MS (70 eV): m/z (\%) $326\left(\mathrm{M}^{+}+1,100\right) ; 307$ (7). Anal. Calcd for $\mathrm{C}_{21} \mathrm{H}_{27} \mathrm{NO}_{2}$ : C 77.50; H 8.36; $\mathrm{N} 4.30$. Found: $\mathrm{C} 77.68 ; \mathrm{H} \mathrm{8.45;} \mathrm{N} \mathrm{4.18.}$

\section{2-(1-Benzyl-3,3-dimethylazetidin-2-yl)-2-methoxyethanol 12c}

Colourless oil, 61\% yield TLC Rf $0.2\left(\mathrm{CH}_{2} \mathrm{Cl}_{2} / \mathrm{MeOH} 33 / 1\right) .{ }^{1} \mathbf{H}$ NMR (300 MHz, $\left.\mathrm{CDCl}_{3}\right): \delta 1.05$ and $1.23(6 \mathrm{H}, 2 \times \mathrm{s}), 2.50(1 \mathrm{H}, \mathrm{d}, J=6.6 \mathrm{~Hz}), 2.94(1 \mathrm{H}, \mathrm{d}, J=9.4 \mathrm{~Hz})$, $2.98(1 \mathrm{H}, \mathrm{d}, J=6.6 \mathrm{~Hz}), 3.35(1 \mathrm{H}, \mathrm{d}, J=13.2 \mathrm{~Hz}), 3.36(1 \mathrm{H}, \mathrm{d}, J=11.5 \mathrm{~Hz}), 3.45-3.50$ $(1 \mathrm{H}, \mathrm{m}), 3.54(3 \mathrm{H}, \mathrm{s}), 3.70(1 \mathrm{H}, \mathrm{d} \times \mathrm{d}, J=11.5 \mathrm{~Hz}, J=3.3 \mathrm{~Hz}), 4.13(1 \mathrm{H}, \mathrm{d}, J=13.2$ $\mathrm{Hz}), 7.19-7.33(5 \mathrm{H}, \mathrm{m}) .{ }^{13} \mathbf{C}$ NMR $\left(75 \mathrm{MHz}, \mathrm{CDCl}_{3}\right): \delta 22.7,28.1,33.8,59.0,61.6,63.4$, $64.9,72.9,83.3,126.8,128.2,128.5,128.7,138.4$. IR $\left(\mathrm{NaCl}, \mathrm{cm}^{-1}\right): v_{\mathrm{OH}}=3412 ; v_{\max }=$ 2952, 2929, 2870, 2822, 1465, 1454, 1367, 1102, 1070, 1029. MS (70 eV): m/z (\%) 250 $\left(\mathrm{M}^{+}+1,100\right)$. Anal. Calcd for $\mathrm{C}_{15} \mathrm{H}_{23} \mathrm{NO}_{2}$ : C 72.25; H 9.30; N 5.62. Found: C 72.42; H 9.22; N 5.69. 


\section{2-(1-Allyl-3,3-dimethylazetidin-2-yl)-2-benzyloxyethanol 12d}

Colourless oil, 49\% yield TLC Rf $0.2\left(\mathrm{CH}_{2} \mathrm{Cl}_{2} / \mathrm{MeOH} 33 / 1\right) .{ }^{1} \mathbf{H}$ NMR (300 MHz, $\left.\mathrm{CDCl}_{3}\right): \delta 1.05$ and $1.23(6 \mathrm{H}, 2 \times \mathrm{s}), 2.48(1 \mathrm{H}, \mathrm{d}, J=6.9 \mathrm{~Hz}), 2.79(1 \mathrm{H}, \mathrm{d} \times \mathrm{d}, J=13.5 \mathrm{~Hz}$, $J=7.4 \mathrm{~Hz}), 2.92(1 \mathrm{H}, \mathrm{d}, J=9.6 \mathrm{~Hz}), 3.07(1 \mathrm{H}, \mathrm{d}, J=6.9 \mathrm{~Hz}), 3.32-3.38(1 \mathrm{H}, \mathrm{m}), 3.61-$ $3.77(3 \mathrm{H}, \mathrm{m}), 4.66(1 \mathrm{H}, \mathrm{d}, J=11.3 \mathrm{~Hz}), 4.72(1 \mathrm{H}, \mathrm{d}, J=11.3 \mathrm{~Hz}), 5.03-5.18(2 \mathrm{H}, \mathrm{m})$, 5.72-5.85 (1H, m), 7.21-7.36 (5H, m). ${ }^{13} \mathbf{C}$ NMR $\left(75 \mathrm{MHz}\right.$, ref $\left.=\mathrm{CDCl}_{3}\right): \delta 22.8,28.4$, $33.9,61.8,62.7,64.8,73.0,73.2,81.8,117.3,126.9,127.3,127.7,127.9,128.4,128.5$, 134.6, 138.8. IR $\left(\mathrm{NaCl}, \mathrm{cm}^{-1}\right): v_{\mathrm{OH}}=3391 ; v_{\max }=2954,2926,2869,1465,1455,1100$, 1062, 1028. MS (70 eV): m/z (\%) $276\left(\mathrm{M}^{+}+1,100\right) ; 257$ (6). Anal. Calcd for $\mathrm{C}_{17} \mathrm{H}_{25} \mathrm{NO}_{2}$ : C 74.14; H 9.15; N 5.09. Found: C 74.01; H 9.27; N 5.03.

\section{2-Benzyloxy-2-(1-cyclohexyl-3,3-dimethylazetidin-2-yl)ethanol 12e}

Colourless oil, 53\% yield TLC Rf $0.2\left(\mathrm{CH}_{2} \mathrm{Cl}_{2} / \mathrm{MeOH} 33 / 1\right) .{ }^{1} \mathbf{H}$ NMR (300 MHz, $\left.\mathrm{CDCl}_{3}\right): \delta 1.03$ and $1.13(6 \mathrm{H}, 2 \times \mathrm{s}), 1.57-1.91(10 \mathrm{H}, \mathrm{m}), 2.12-2.23(1 \mathrm{H}, \mathrm{m}), 2.67(1 \mathrm{H}, \mathrm{d}, J$ $=7.0 \mathrm{~Hz}), 3.09(1 \mathrm{H}, \mathrm{d}, J=7.0 \mathrm{~Hz}), 3.32(1 \mathrm{H}, \mathrm{d}, J=7.2 \mathrm{~Hz}), 3.58-3.66(1 \mathrm{H}, \mathrm{m}), 3.73-$ $3.80(1 \mathrm{H}, \mathrm{m}), 3.97(1 \mathrm{H}, \mathrm{d} \times \mathrm{d}, J=11.0 \mathrm{~Hz}, J=6.6 \mathrm{~Hz}), 4.58(1 \mathrm{H}, \mathrm{d}, J=11.8 \mathrm{~Hz}), 4.65$ $(1 \mathrm{H}, \mathrm{d}, J=11.8 \mathrm{~Hz}), 7.26-7.36(5 \mathrm{H}, \mathrm{m}) .{ }^{13} \mathbf{C} \mathbf{N M R}\left(75 \mathrm{MHz}, \mathrm{CDCl}_{3}\right): \delta 25.1,25.3,26.0$ 27.7, 28.6, 29.9, 31.5, 33.8, 62.4, 62.9, 65.3, 71.0, 72.6, 78.8, 127.6, 127.6, 127.7, 128.5, 128.6, 138.7. IR $\left(\mathrm{NaCl}, \mathrm{cm}^{-1}\right): v_{\mathrm{OH}}=3392 ; v_{\max }=2929,2854,1464,1453,1369,1099$, 1060, 1028. MS $(70 \mathrm{eV}): \mathrm{m} / \mathrm{z}(\%) 318\left(\mathrm{M}^{+}+1,100\right)$. Anal. Calcd for $\mathrm{C}_{20} \mathrm{H}_{31} \mathrm{NO}_{2}$ : C 75.67; H 9.84; N 4.41. Found: C 75.83; H 9.77; N 4.31. 


\section{Cis-3-benzyloxy-4-[(2-bromo-1,1-dimethyl)ethyl]-1-t-butylazetidine 13a}

White crystals, 5\% yield, TLC Rf 0.3 (petroleum ether/ethyl acetate 9/1), Mp. $63{ }^{\circ} \mathrm{C}{ }^{1} \mathbf{H}$ NMR (300 MHz, $\left.\mathrm{CDCl}_{3}\right): \delta 0.97(9 \mathrm{H}, \mathrm{s}), 1.14$ and $1.24(6 \mathrm{H}, 2 \times \mathrm{s}), 3.30(2 \mathrm{H}, \mathrm{m}), 3.45$ $(1 \mathrm{H}, \mathrm{m}), 3.53(1 \mathrm{H}, \mathrm{d}, J=9.6 \mathrm{~Hz}), 3.89(1 \mathrm{H}, \mathrm{d}, J=9.6 \mathrm{~Hz}), 4.33(1 \mathrm{H}, \mathrm{d}, J=12.1 \mathrm{~Hz})$ $4.34(1 \mathrm{H}, \mathrm{m}), 4.40(1 \mathrm{H}, \mathrm{d}, J=12.1 \mathrm{~Hz}), 7.19-7.39(5 \mathrm{H}, \mathrm{m}) .{ }^{13} \mathbf{C}$ NMR $(75 \mathrm{MHz}$, ref $=$ $\left.\mathrm{CDCl}_{3}\right): \delta 23.8,24.5,26.7,38.4,46.4,52.5,53.8,68.4,71.1,71.8,127.1,127.4,127.7$, 127.7, 127.9, 128.5, 128.6, 138.4. IR $\left(\mathrm{KBr}, \mathrm{cm}^{-1}\right): v_{\max }=2973,2930,2874,2892,2811$, 1454, 1369, 1360, 1226, 1200, 1104, 1011. MS (70 eV): m/z (\%) 356/4 (M+1, 100); 275 (7). Anal. Calcd for $\mathrm{C}_{18} \mathrm{H}_{28}$ BrNO: $\mathrm{C} 61.02 ; \mathrm{H} 7.97$; N 3.95. Found: $\mathrm{C} 60.79 ; \mathrm{H}$ 7.80; $\mathrm{N}$ 4.14.

\section{Cis-1-benzyl-3-benzyloxy-4-[(2-bromo-1,1-dimethyl)ethyl]azetidine 13b}

Colourless oil, 4\% yield, TLC Rf 0.3 (petroleum ether/ethyl acetate 9/1). ${ }^{1} \mathbf{H}$ NMR (300 $\left.\mathrm{MHz}_{\mathrm{CDCl}}\right): \delta 1.19$ and $1.22(6 \mathrm{H}, 2 \times \mathrm{s}), 2.86(1 \mathrm{H}, \mathrm{d} \times \mathrm{d}, J=9.2 \mathrm{~Hz}, J=6.5 \mathrm{~Hz}), 3.35$ $(1 \mathrm{H}, \mathrm{d} \times \mathrm{d}, J=9.2 \mathrm{~Hz}, J=3.0 \mathrm{~Hz}), 3.43(1 \mathrm{H}, \mathrm{d}, J=7.2 \mathrm{~Hz}), 3.43(1 \mathrm{H}, \mathrm{d}, J=13.4 \mathrm{~Hz})$, $3.56(1 \mathrm{H}, \mathrm{d}, J=9.9 \mathrm{~Hz}), 3.83(1 \mathrm{H}, \mathrm{d}, J=9.9 \mathrm{~Hz}), 3.99(1 \mathrm{H}, \mathrm{d}, J=13.4 \mathrm{~Hz}), 4.26(1 \mathrm{H}, \mathrm{d}$, $J=12.1 \mathrm{~Hz}), 4.35(1 \mathrm{H}, \mathrm{d} \times \mathrm{d} \times \mathrm{d}, J=7.2 \mathrm{~Hz}, J=6.5 \mathrm{~Hz}, J=3.0 \mathrm{~Hz}), 4.46(1 \mathrm{H}, \mathrm{d}, J=12.1$ $\mathrm{Hz}), 7.20-7.36(10 \mathrm{H}, \mathrm{m}) .{ }^{13} \mathbf{C}$ NMR $\left(75 \mathrm{MHz}\right.$, ref $\left.=\mathrm{CDCl}_{3}\right): \delta 24.1,24.4,38.3,45.9$, 58.1, 63.6, 70.9, 72.6, 74.7, 127.0, 127.4, 127.6, 128.3, 128.5, 128.8, 138.3, 139.0. IR $\left(\mathrm{NaCl}, \mathrm{cm}^{-1}\right): v_{\max }=2962,2929,2867,1495,1454,1362,1166,1117,1057,1028 . \mathbf{M S}$ $(70 \mathrm{eV}): \mathrm{m} / \mathrm{z}(\%)$ 388/390 $\left(\mathrm{M}^{+}+1,100\right) ; 341(6) ; 328$ (18); 327 (84); $311(24) ; 310(28)$; 309 (88). Anal. Calcd for $\mathrm{C}_{21} \mathrm{H}_{26}$ BrNO: C 64.95; H 6.75; N 3.61. Found: C 64.79; H 6.76; N 3.75. 


\section{Cis-1-benzyl-4-[(2-bromo-1,1-dimethyl)ethyl]-3-methoxyazetidine 13c}

Colourless oil, $1 \%$ yield, TLC Rf 0.3 (petroleum ether/ethyl acetate 9/1). ${ }^{1} \mathbf{H}$ NMR (300 $\left.\mathrm{MHz}, \mathrm{CDCl}_{3}\right): \delta 1.14$ and $1.18(6 \mathrm{H}, 2 \times \mathrm{s}), 2.81(1 \mathrm{H}, \mathrm{d} \times \mathrm{d}, J=9.1 \mathrm{~Hz}, J=6.3 \mathrm{~Hz}), 3.16$ $(3 \mathrm{H}, \mathrm{s}), 3.27(1 \mathrm{H}, \mathrm{d} \times \mathrm{d} \times \mathrm{d}, J=9.1 \mathrm{~Hz}, J=2.5 \mathrm{~Hz}, J=1.1 \mathrm{~Hz}), 3.34-3.38(1 \mathrm{H}, \mathrm{m}), 3.39$ $(1 \mathrm{H}, \mathrm{d}, J=13.2 \mathrm{~Hz}), 3.55(1 \mathrm{H}, \mathrm{d}, J=9.7 \mathrm{~Hz}), 3.80(1 \mathrm{H}, \mathrm{d}, J=9.7 \mathrm{~Hz}), 3.99(1 \mathrm{H}, \mathrm{d}, J=$ 13.2 Hz), 4.08-4.13 $(1 \mathrm{H}, \mathrm{d} \times \mathrm{t}, J=6.3 \mathrm{~Hz}, J=2.5 \mathrm{~Hz}), 7.19-7.33(5 \mathrm{H}, \mathrm{m}) .{ }^{13} \mathbf{C}$ NMR (75 $\left.\mathrm{MHz}, \mathrm{CDCl}_{3}\right): \delta 23.9,24.4,38.1,45.8,56.2,57.5,63.6,74.8,74.8,127.0,128.3,128.5$, 138.7. IR $\left(\mathrm{NaCl}, \mathrm{cm}^{-1}\right): v_{\max }=2964,2930,2829,1453,1382,1366,1243,1192,1182$, 1157, 1117, 1059. MS (70 eV): m/z (\%) 312/4 (M+1, 59); 235 (6); 234 (13); 233 (100). Anal. Calcd for $\mathrm{C}_{15} \mathrm{H}_{22}$ BrNO: C 57.70; H 7.10; N 4.49. Found: C 57.84; H 7.02; N 4.45.

\section{Cis-1-allyl-3-benzyloxy-4-[(2-bromo-1,1-dimethyl)ethyl]azetidine 13d}

Colourless oil, 3\% yield, TLC Rf 0.3 (petroleum ether/ethyl acetate 9/1). ${ }^{1} \mathbf{H}$ NMR (300 $\left.\mathrm{MHz}, \mathrm{CDCl}_{3}\right): \delta 1.16$ and $1.17(6 \mathrm{H}, 2 \times \mathrm{s}), 2.88(1 \mathrm{H}, \mathrm{d} \times \mathrm{d}, J=9.1 \mathrm{~Hz}, J=6.3 \mathrm{~Hz}), 2.91$ $(1 \mathrm{H}, \mathrm{d} \times \mathrm{d}, J=13.5 \mathrm{~Hz}, J=7.2 \mathrm{~Hz}), 3.28(1 \mathrm{H}, \mathrm{d} \times \mathrm{d}, J=7.2 \mathrm{~Hz}, J=1.1 \mathrm{~Hz}), 3.36-3.41(2 \mathrm{H}$, m), $3.52(1 \mathrm{H}, \mathrm{d}, J=9.9 \mathrm{~Hz}), 3.80(1 \mathrm{H}, \mathrm{d}, J=9.9 \mathrm{~Hz}), 4.28(1 \mathrm{H}, \mathrm{d}, J=12.3 \mathrm{~Hz}), 4.29-$ $4.34(1 \mathrm{H}, \mathrm{m}), 4.50(1 \mathrm{H}, \mathrm{d}, J=12.3 \mathrm{~Hz}), 5.06-5.20(2 \mathrm{H}, \mathrm{m}), 5.73-5.86(1 \mathrm{H}, \mathrm{m}), 7.23-7.39$ $(5 \mathrm{H}, \mathrm{m}) .{ }^{13} \mathrm{C}$ NMR $\left(75 \mathrm{MHz}\right.$, ref $\left.=\mathrm{CDCl}_{3}\right): \delta 24.1,24.3,38.1,45.9,57.6,62.1,70.9$, $72.8,74.4,117.2,127.5,127.6,128.5,135.0,138.2 . \mathbf{I R}\left(\mathrm{NaCl}, \mathrm{cm}^{-1}\right): v_{\max }=2927,2867$, 1455, 1362, 1187, 1167, 1114, 1049, 1028. MS (70 eV): m/z (\%) 338/340 (M+1, 100); 277/279 (8); 260/262 (8); 259/61 (32). Anal. Calcd for $\mathrm{C}_{17} \mathrm{H}_{24} \mathrm{BrNO}$ : C 60.36; H 7.15; N 4.14. Found: C 60.21; H 7.33; N 4.28. 


\section{Cis-4-[(2-bromo-1,1-dimethyl)ethyl]-1-cyclohexyl-3-methoxyazetidine 13e}

Colourless oil, 5\% yield, TLC Rf 0.3 (petroleum ether/ethyl acetate 9/1). ${ }^{1} \mathbf{H}$ NMR (300 $\left.\mathrm{MHz}, \mathrm{CDCl}_{3}\right): \delta 1.16$ and $1.22(6 \mathrm{H}, 2 \times \mathrm{s}), 1.57-1.88(10 \mathrm{H}, \mathrm{m}), 2.36-2.42(1 \mathrm{H}, \mathrm{m}), 3.26-$

$3.37(2 \mathrm{H}, \mathrm{m}), 3.30(1 \mathrm{H}, \mathrm{d}, J=7.2 \mathrm{~Hz}), 3.53(1 \mathrm{H}, \mathrm{d}, J=9.6 \mathrm{~Hz}), 3.84(1 \mathrm{H}, \mathrm{d}, J=9.6 \mathrm{~Hz})$, $4.48(1 \mathrm{H}, \mathrm{d}, J=11.6 \mathrm{~Hz}), 4.46-4.52(1 \mathrm{H}, \mathrm{m}), 4.64(1 \mathrm{H}, \mathrm{d}, J=11.6 \mathrm{~Hz}), 7.23-7.42(5 \mathrm{H}$, m). ${ }^{13} \mathbf{C}$ NMR $\left(75 \mathrm{MHz}, \mathrm{CDCl}_{3}\right): \delta 26.0,26.1,26.5,28.5,29.2,36.4,46.0,53.2,63.6$, 69.1, 70.4, 73.3, 127.7, 127.8, 128.5, 138.3. IR $\left(\mathrm{NaCl}, \mathrm{cm}^{-1}\right): v_{\max }=2928,2854,1453$, 1382, 1364, 1255, 1210, 1172, 1109, 1028. MS (70 eV): m/z (\%) 380/2 $\left(\mathrm{M}^{+}+1,75\right)$. Anal. Calcd for $\mathrm{C}_{20} \mathrm{H}_{30} \mathrm{BrNO}$ : C 63.15; H 7.95; N 3.68. Found: C 63.25; H 7.85; N 3.58.

\section{General procedure for the synthesis of trans-4-alkylamino-3-}

\section{alkoxytetrahydrofurans 9}

As an example, the synthesis of trans-4-benzylamino-3-benzyloxy-5,5-dimethyloxolane 9a is described. To a slurry of $\mathrm{AlCl}_{3}(0.21 \mathrm{~g}, 1.61 \mathrm{mmol})$ and $30 \mathrm{ml}$ of dry diethyl ether was added 2-benzyloxy-2-(1-tert-butyl-3,3-dimethylaziridin-2-yl)ethanol 2a (0.5g, 1.61 mmol) and the resulting reaction mixture was refluxed for 48 hours. Then, $30 \mathrm{ml}$ of water was added and the mixture was extracted three times with $30 \mathrm{ml}$ of diethyl ether. The combined organic fractions were dried $\left(\mathrm{MgSO}_{4}\right)$ and after filtration and evaporation of the solvent, the crude reaction mixture was obtained. Further purification was performed by flash chromatography over silica gel. 


\section{Trans-4-benzylamino-3-benzyloxy-5,5-dimethyloxolane 9a}

Colourless oil, 55\% yield, TLC Rf $0.35\left(\mathrm{CH}_{2} \mathrm{Cl}_{2} / \mathrm{MeOH} 33 / 1\right) .{ }^{1} \mathbf{H}$ NMR $(300 \mathrm{MHz}$, $\left.\mathrm{CDCl}_{3}\right): \delta 1.18$ and $1.34(6 \mathrm{H}, 2 \times \mathrm{s}), 3.03(1 \mathrm{H}, \mathrm{d}, J=4.7 \mathrm{~Hz}), 3.74-3.78(1 \mathrm{H}, \mathrm{m}), 3.82(1 \mathrm{H}$, d, $J=13.2 \mathrm{~Hz}), 3.89(1 \mathrm{H}, \mathrm{d}, J=13.2 \mathrm{~Hz}), 3.96-4.05(2 \mathrm{H}, \mathrm{m}), 4.49(1 \mathrm{H}, \mathrm{d}, J=11.8 \mathrm{~Hz})$, $4.53(1 \mathrm{H}, \mathrm{d}, J=11.8 \mathrm{~Hz}), 7.22-7.38(10 \mathrm{H}, \mathrm{m}){ }^{\mathbf{1 3}} \mathbf{C} \mathbf{N M R}\left(75 \mathrm{MHz}, \mathrm{CDCl}_{3}\right): \delta 21.8,27.7$, 52.6, 69.0, 71.0, 72.0, 81.8, 85.6, 126.9, 127.1, 127.8, 128.1, 128.4, 128.4, 138.0, 140.3. IR $\left(\mathrm{NaCl}, \mathrm{cm}^{-1}\right): v_{\max }=3426,2972,2929,2868,1496,1454,1366 . \mathbf{M S}(70 \mathrm{eV}) \mathrm{m} / \mathrm{z}(\%)$ : $312\left(\mathrm{M}^{+}+1,100\right)$. Anal. Calcd for $\mathrm{C}_{20} \mathrm{H}_{25} \mathrm{NO}_{2}$ : C 77.14; $\mathrm{H}$ 8.09; $\mathrm{N}$ 4.50. Found: C 77.29; H 8.19; N 4.38 .

\section{Trans-4-benzylamino-3-methoxy-5,5-dimethyloxolane 9b}

Colourless oil, 62\% yield TLC Rf $0.2\left(\mathrm{CH}_{2} \mathrm{Cl}_{2} / \mathrm{MeOH} 33 / 1\right) .{ }^{1} \mathbf{H}$ NMR $(300 \mathrm{MHz}$, $\left.\mathrm{CDCl}_{3}\right): \delta 1.20$ and $1.31(6 \mathrm{H}, 2 \times \mathrm{s}), 2.35(1 \mathrm{H}$, broad s $), 2.93(1 \mathrm{H}, \mathrm{d}, J=4.7 \mathrm{~Hz}), 3.31(3 \mathrm{H}$, s), $3.72(1 \mathrm{H}, \mathrm{d} \times \mathrm{d}, J=9.6 \mathrm{~Hz}, J=4.4 \mathrm{~Hz}), 3.83(1 \mathrm{H}, \mathrm{d}, J=13.1 \mathrm{~Hz}), 3.80-3.85(1 \mathrm{H}, \mathrm{m})$, $3.93(1 \mathrm{H}, \mathrm{d}, J=13.1 \mathrm{~Hz}), 4.03(1 \mathrm{H}, \mathrm{d} \times \mathrm{d}, J=9.6 \mathrm{~Hz}, J=6.1 \mathrm{~Hz}), 7.22-7.39(5 \mathrm{H}, \mathrm{m}){ }^{\mathbf{1 3}} \mathbf{C}$ NMR $\left(67.8 \mathrm{MHz} \mathrm{CDCl}_{3}\right): \delta 21.7,27.7,52.5,57.4,68.7,70.7,81.8,87.5,127.1,128.2$ 128.4, 139.8. IR $\left(\mathrm{NaCl}, \mathrm{cm}^{-1}\right): v_{\max }=3338,2973,2930,1660,1604,1495,1454,1367$. MS $(70 \mathrm{eV}) \mathrm{m} / \mathrm{z}(\%): 236\left(\mathrm{M}^{+}+1,100\right)$. Anal. Calcd for $\mathrm{C}_{14} \mathrm{H}_{21} \mathrm{NO}_{2}: \mathrm{C} 71.46 ; \mathrm{H} 8.99 ; \mathrm{N}$ 5.95. Found: C 71.36; H 9.16; N 5.84.

\section{Trans-4-allylamino-3-benzyloxy-5,5-dimethyloxolane 9c}

Colourless oil, 64\% yield TLC Rf $0.2\left(\mathrm{CH}_{2} \mathrm{Cl}_{2} / \mathrm{MeOH} 33 / 1\right) .{ }^{1} \mathbf{H}$ NMR (300 MHz, $\left.\mathrm{CDCl}_{3}\right): \delta 1.15$ and $1.35(6 \mathrm{H}, 2 \times \mathrm{s}), 3.00(1 \mathrm{H}, \mathrm{d}, J=4.4 \mathrm{~Hz}), 3.24-3.39(2 \mathrm{H}, \mathrm{m}), 3.73-3.78$ 
$(1 \mathrm{H}, \mathrm{m}), 3.91-4.03(2 \mathrm{H}, \mathrm{m}), 4.52(2 \mathrm{H}, \mathrm{s}), 5.08-5.23(2 \mathrm{H}, \mathrm{m}), 5.84-5.97(1 \mathrm{H}, \mathrm{m}), 7.27-$ $7.44(5 \mathrm{H}, \mathrm{m}) .{ }^{13} \mathbf{C}$ NMR $\left(67.8 \mathrm{MHz}, \mathrm{CDCl}_{3}\right): \delta 21.7,27.7,51.0,68.9,70.7,71.9,81.6$, 85.5, 116.0, 127.7, 127.7, 128.4, 136.8, 138.1. IR $\left(\mathrm{NaCl}, \mathrm{cm}^{-1}\right): v_{\max }=3447,3332,3065$, 3031, 2973, 2929, 2868, 1643, 1497, 1455, 1366. MS (70 eV) m/z (\%): $262\left(\mathrm{M}^{+}+1,100\right)$. Anal. Calcd for $\mathrm{C}_{16} \mathrm{H}_{23} \mathrm{NO}_{2}$ : C 73.53; H 8.87; N 5.36. Found: C 73.59; H 8.98; N 5.49.

\section{Trans-4-allylamino-3-benzyloxy-5,5-dimethyloxolane 9d}

Colourless oil, 53\% yield TLC Rf $0.2\left(\mathrm{CH}_{2} \mathrm{Cl}_{2} / \mathrm{MeOH} 33 / 1\right) .{ }^{1} \mathrm{H}$ NMR $(300 \mathrm{MHz}$, $\left.\mathrm{CDCl}_{3}\right): \delta 1.16$ and $1.33(6 \mathrm{H}, 2 \times \mathrm{s}), 1.95(1 \mathrm{H}$, broad s), $2.91(1 \mathrm{H}, \mathrm{d}, J=4.7 \mathrm{~Hz}), 3.26-3.42$ $(2 \mathrm{H}, \mathrm{m}), 3.34(3 \mathrm{H}, \mathrm{s}), 3.70-3.78(2 \mathrm{H}, \mathrm{m}), 4.00-4.07(1 \mathrm{H}, \mathrm{m}), 5.10-5.25(2 \mathrm{H}, \mathrm{m}), 5.86-$ $5.99(1 \mathrm{H}, \mathrm{m}) .{ }^{13} \mathrm{C}$ NMR $\left(67.8 \mathrm{MHz}\right.$, ref $\left.=\mathrm{CDCl}_{3}\right): \delta 21.7,27.8,51.1,57.6,68.8,70.7$, 81.8, 87.7, 116.3, 136.7. IR $\left(\mathrm{NaCl}, \mathrm{cm}^{-1}\right): v_{\max }=3402,2972,2929,1644,1461,1368 . \mathbf{M S}$ $(70 \mathrm{eV}) \mathrm{m} / \mathrm{z}(\%): 185\left(\mathrm{M}^{+}, 10\right) ; 112$ (100); 96 (50). Anal. Calcd for $\mathrm{C}_{10} \mathrm{H}_{19} \mathrm{NO}_{2}$ : C 64.83; H 10.34; N 7.56. Found: C 64.64; H 10.49; N 7.77.

\section{Trans-3-benzyloxy-4-tert-butylamino-5,5-dimethyloxolane 9e}

Colourless oil, 56\% yield, TLC Rf 0.22 (petroleum ether/ethyl acetate 3/2). ${ }^{1} \mathbf{H}$ NMR (300 MHz, $\left.\mathrm{CDCl}_{3}\right): \delta 1.08$ and $1.29(6 \mathrm{H}, 2 \times \mathrm{s}), 1.10(9 \mathrm{H}, \mathrm{s}), 3.03(1 \mathrm{H}, \mathrm{d}, J=4.4 \mathrm{~Hz})$, 3.75-3.80 (2H, m), $3.93(1 \mathrm{H}, \mathrm{d} \times \mathrm{d}, J=11.0 \mathrm{~Hz}, J=6.9 \mathrm{~Hz}), 4.43(1 \mathrm{H}, \mathrm{d}, J=11.4 \mathrm{~Hz})$, $4.48(1 \mathrm{H}, \mathrm{d}, J=11.4 \mathrm{~Hz}), 7.24-7.39(5 \mathrm{H}, \mathrm{m}) .{ }^{13} \mathbf{C} \mathbf{~ N M R}\left(75 \mathrm{MHz}, \mathrm{CDCl}_{3}\right): \delta 21.5,27.1$, $30.3,50.7,66.2,68.7,72.3,81.8,88.3,127.6,127.8,128.3,138.1 . \mathbf{I R}\left(\mathrm{NaCl}, \mathrm{cm}^{-1}\right): v_{\max }=$ 3430, 2968, 2931, 2868, 1715, 1497, 1455, 1389, 1364. MS (70 eV) m/z (\%): 278 
$\left(\mathrm{M}^{+}+1,100\right)$. Anal. Calcd for $\mathrm{C}_{17} \mathrm{H}_{27} \mathrm{NO}_{2}$ : C 73.61; H 9.81; N 5.05. Found: $\mathrm{C} 73.65 ; \mathrm{H}$

9.66; N 4.89. 